\title{
Télescope
}

Revue d'analyse comparée en administration publique

\section{Collusion et corruption dans les administrations : les vérificateurs généraux seraient-ils condamnés à n'être que des tigres de papier?}

\author{
Bertrand Malsch, Danielle Morin et Marie-Soleil Tremblay
}

Volume 18, numéro 3, automne 2012

URI : https://id.erudit.org/iderudit/1013781ar

DOI : https://doi.org/10.7202/1013781ar

Aller au sommaire du numéro

Éditeur(s)

L’Observatoire de l'administration publique

ISSN

1203-3294 (imprimé)

1929-3348 (numérique)

Découvrir la revue

Citer cet article

Malsch, B., Morin, D. \& Tremblay, M.-S. (2012). Collusion et corruption dans les administrations : les vérificateurs généraux seraient-ils condamnés à n'être que des tigres de papier? Télescope, 18(3), 173-180.

https://doi.org/10.7202/1013781ar d'utilisation que vous pouvez consulter en ligne. 


\title{
Débat
}

\section{COLLUSION ET CORRUPTION DANS LES ADMINISTRATIONS : LES VÉRIFICATEURS GÉNÉRAUX SERAIENT-ILS CONDAMNÉS À N'ÊTRE QUE DES TIGRES DE PAPIER?}

\author{
Par Bertrand Malsch, Professeur adjoint, HEC Montréal • bertrand.malsch@hec.ca \\ Danielle Morin, Professeure titulaire, HEC Montréal • danielle.morin@hec.ca \\ Et Marie-Soleil Tremblay, Professeure, École nationale d'administration publique \\ - marie-soleil.tremblay@enap.ca
}

Pour citer cet article : Malsch, B., D. Morin et M.-S. Tremblay (2012). « Collusion et corruption dans les administrations : les vérificateurs généraux seraient-ils condamnés à n'être que des tigres de papier? », Télescope, vol. 18, n³, p. 173-180.

$\mathrm{C}$ réée le 9 novembre 2011 après valses-hésitations du gouvernement Charest, la commission Charbonneau, chargée d'enquêter sur l'octroi et la gestion des contrats publics dans l'industrie de la construction, a permis jusqu'à présent de révéler en plein jour les allusions de collusion et la corruption ayant eu cours dans certaines administrations municipales : fonctionnaires corrompus, parties de golf avec des dirigeants de la mafia, financement occulte de partis politiques, dilapidation de fonds publics. Les maires des plus grandes villes du Québec, Montréal et Laval, sont mis directement en cause par des témoins devant la Commission.

Il serait naïf d'imaginer que la province de Québec soit le seul lieu frappé par ces relations troubles entre administrations publiques, organisations mafieuses et pouvoirs politiques. Dunleavy et Hood (1994) font ainsi l'observation suivante : « The basic problems of public management - namely human error, system failures, misdirected programmes, fraud or corruption and bad intentions are omnipresent. "Cependant, la constatation de ce principe de réalité ne doit pas nous interdire en tant que chercheurs et en tant qu'intellectuels de réfléchir publiquement aux causes de ces dysfonctionnements, d'apporter des éléments de solution et de contribuer aux changements (Cooper, 2005; Malsch et Guénin-Paracini, à paraître; Neu, Cooper et Everett, 2001). En d'autres termes, ni la naïveté ni le fatalisme ne doivent l'emporter sur la volonté de réforme et l'exigence démocratique de débattre en société (Arendt, 1979).

Notre expertise, notre position de libres penseurs et notre désir d'étendre le débat au-delà de simples constats de résignation et d'impuissance nous ont ainsi poussés à exprimer dans les colonnes d'un grand quotidien du Québec un point de vue sur le rôle et la responsabilité des vérificateurs généraux au regard de la prévention et de la détection de la corruption dans les administrations. En effet, si ces derniers ont notamment pour mandat de s'assurer que les ressources publiques sont gérées dans un souci d'économie, d'efficience et d'efficacité (Morin, 2002), comment expliquer que ces pratiques mafieuses, apparemment connues de tous 
au sein des hôtels de ville, soient passées pendant tant d'années au travers de leurs filets de contrôle? En réponse à cette interrogation, le vérificateur général de la Ville de Montréal et ses adjoints ont publié à leur tour une réplique pour réfuter leur mise en cause, défendre le bilan et pointer les limitations de leur mandat.

Nous pensons que la publication de notre point de vue et de celui du vérificateur général de la Ville de Montréal présente une contribution hautement pertinente à ce numéro spécial de Télescope. L'écriture d'une tribune d'opinion est un travail de synthèse et d'argumentation. Réunir dans un même article ces deux opinions permet donc de camper très efficacement certains enjeux fondamentaux liés aux méthodes de travail et à l'indépendance des vérificateurs. Par ailleurs, la superposition de ces deux points de vue permet également de mettre en perspective des conceptions différentes et de les problématiser.

La suite de cet essai s'organise de la manière suivante. La première partie restitue notre point de vue publié dans La Presse, la seconde présente la réponse du vérificateur général de la Ville de Montréal et la troisième discute l'opposition de ces points de vue et les situe dans une perspective critique en s'interrogeant sur la capacité des vérificateurs généraux de contribuer à la prévention, à la détection et, ultimement, à la correction de la fraude et de la corruption dans les administrations publiques.

\section{Où étaient les vérificateurs généraux ?}

La commission Charbonneau est en train de faire en partie leur travail.

Ont-ils le pouvoir et l'autorité nécessaire pour mener leurs enquêtes face à des puissances mafieuses?

Confronté au grand Barnum de déclarations, de témoignages, d'allégations et d'accusations qui rythment presque quotidiennement les travaux de la commission Charbonneau, le Québec, abasourdi, semble découvrir chaque jour davantage un système de passe-droit institutionnalisé dans les rouages d'administrations municipales. Ces révélations risquent fort de venir alimenter chez les citoyens un sentiment de grande frustration et de cynisme vis-à-vis du personnel politique de la province. Ce serait cependant une erreur de faire des politiciens les seuls boucs émissaires de cette crise de confiance.

Ce serait oublier, d'une part, que les maires et les ministres qui nous gouvernent ont été élus - et dans bien des cas réélus - démocratiquement par la population. Ce serait oublier, d'autre part, que les municipalités et le gouvernement agissent en théorie sous l'oeil vigilant et indépendant de vérificateurs généraux dont la mission est précisément de contrôler l'utilisation des ressources publiques et de s'assurer qu'elles sont gérées en conformité avec les lois et un souci d'économie, d'efficience et d'efficacité.

Chaque année, les vérificateurs généraux de la Ville de Montréal, de la Ville de Laval ou du gouvernement du Québec produisent plusieurs centaines de pages

Bertrand Malsch, Danielle Morin et Marie-Soleil Tremblay (2012), « Où étaient les vérificateurs généraux? », La Presse, Section Débats, 29 octobre, p. A-18. 
pour rendre compte de leurs audits et faire part de leurs recommandations. En septembre 2009, le vérificateur général de la Ville de Montréal rédigeait ainsi un rapport remarqué dans lequel il mettait en évidence différentes zones d'ombre à forte odeur de collusion entourant l'attribution du marché des compteurs d'eau de la Ville de Montréal. On sait ce qu'il est advenu de ce rapport et des nombreux coups qui ont été portés par la suite contre Jacques Bergeron pour tenter de le décrédibiliser.

Le rôle d'un vérificateur, qu'il s'agisse du secteur public ou du secteur privé, consiste à produire de la confiance entre l'entité qu'il contrôle et le public qui en dépend. Lorsqu'il échoue, les conséquences sont graves. La disparition brutale du cabinet Arthur Andersen impliqué dans l'affaire Enron est là pour en témoigner. La confiance est un élément fondamental, non seulement pour le climat des affaires, mais également pour l'harmonie sociale. Tout ce qui altère le lien social entre le gouvernement et les citoyens est une atteinte sérieuse au contrat démocratique.

Qu'il ait fallu ainsi attendre une commission d'enquête publique pour faire toute la lumière sur la gestion illégale et inefficace des fonds publics dans le domaine de la construction - c'est-à-dire pour faire en partie le travail attendu des vérificateurs - appelle sans délai un débat public autour des questions suivantes. Les vérificateurs de la province et des municipalités du Québec disposent-ils des ressources humaines et financières nécessaires pour exécuter leur mission? Ont-ils le pouvoir et l'autorité nécessaire pour mener leurs enquêtes face à des puissances mafieuses? Les contrôles internes des administrations publiques sont-ils à ce point défaillants qu'ils rendent la tâche des vérificateurs impossible? Sont-ils suffisamment protégés des pressions du pouvoir politique pour pouvoir assumer leur rôle de chien de garde et ne pas hésiter à bousculer les intérêts les plus établis? Bref, la vérification des comptes et de la gestion des administrations telle qu'elle est pratiquée aujourd'hui est-elle encore le bon outil pour détecter les menaces de fraude et de corruption?

Lorsque les feux des projecteurs médiatiques se seront éteints sur la commission Charbonneau, il reviendra au gouvernement d'en appliquer les recommandations et aux vérificateurs généraux d'en surveiller la mise en œuvre. En conséquence, si les travaux de la Commission devaient se conclure sans avoir sérieusement réfléchi au rôle et aux responsabilités des vérificateurs généraux, il y aurait tout lieu de craindre que la confiance du public ne puisse être pleinement rétablie, non seulement vis-à-vis de ceux qui nous gouvernent, mais également visà-vis de ceux qui les surveillent. 


\section{L'alarme sonnée à plusieurs reprises ${ }^{2}$}

Depuis 2004, nous avons produit plus de 25 rapports sur l'octroi et la gestion des contrats à la Ville de Montréal.

Lorsque nous confirmons la mise en application de nos recommandations à la suite de la découverte de lacunes, notre cycle de vérification prend fin. Si, par la suite, il y avait récidive des lacunes, nous ne pourrions pas détecter cette situation.

Nous tenons à nuancer certaines affirmations émises dans la lettre des professeurs Bertrand Malsch, Danielle Morin et Marie-Soleil Tremblay à la lumière du contexte propre à la fonction du vérificateur général de la Ville de Montréal.

Le mandat conféré aux vérificateurs généraux municipaux en vertu de la Loi sur les cités et villes est de soutenir le conseil municipal dans ses responsabilités de gouverne par le moyen de sa vérification des affaires et comptes de la municipalité. Cette vérification comporte notamment la mesure dans laquelle les ressources sont gérées par l'administration en conformité avec les lois et les règlements ainsi qu'un souci d'économie, d'efficience et d'efficacité.

Le vérificateur général de la Ville de Montréal a-t-il sonné l'alarme en ce qui a trait plus spécifiquement au processus d'octroi et de gestion des contrats relatifs aux travaux de construction? À maintes reprises. Nous avons produit, depuis 2004, plus de 25 rapports de vérification qui touchaient l'octroi et la gestion des contrats. Nous avons également, à quelques reprises, transmis le fruit de nos travaux aux autorités policières, dont à titre d'exemple le dossier du projet Faubourg Contrecoeur.

Par ailleurs, nous ne pouvons passer sous silence, en lien avec les allégations faites à la commission Charbonneau sur le processus d'octroi et de gestion de contrats à la Ville de Montréal, que lorsque nous confirmons la mise en application de nos recommandations à la suite de la découverte de lacunes, notre cycle de vérification prend fin compte tenu de notre mandat. Si, par la suite, pour différentes raisons, il y avait récidive des dites lacunes, nous ne pourrions pas détecter cette situation.

De plus, si une personne décide sciemment, une fois la recommandation mise en place, de la contourner, il nous serait également difficile de découvrir cette situation. En outre, rappelons que le vérificateur général n'a qu'un pouvoir de recommandation. La mise en œuvre ou non des recommandations formulées par le vérificateur général est une prérogative de l'administration.

Dans notre dernier rapport annuel, nous avons fait état qu'environ $5 \%$ des recommandations dites majeures n'avaient pas été mises en application. Nous ne disons pas que toutes ces recommandations concernent nécessairement les allégations faites à la commission Charbonneau, mais cette situation démontre les limites des travaux du vérificateur général.

Nous n'avons pas, à titre de vérificateur général, la capacité et la latitude, comme l'affirment les auteurs, de faire " toute la lumière sur la gestion illégale et

2 Jacques Bergeron, Robert Duquette et Serge Vaillancourt (2012), " L'alarme sonnée à plusieurs reprises », La Presse, Section Débats, 2 novembre, p. A-22. 
inefficace des fonds publics dans le domaine de la construction ». La portée de notre travail et de nos conclusions est limitée par quatre facteurs majeurs.

- Le vérificateur général n'a pas les pouvoirs d'enquête des autorités policières.

- Contrairement à la commission Charbonneau, qui a le pouvoir de contraindre des personnes appartenant à des organisations diverses à venir témoigner à sa tribune, le pouvoir du vérificateur général de la Ville de Montréal se limite à exiger de tout employé de la Ville de lui fournir les informations nécessaires à son travail. Il n'a pas légalement l'autorité nécessaire d'exiger des informations d'élus ou de tiers ainsi que de les contraindre à s'expliquer.

- Une limite inhérente au contrôle interne dans une organisation est la présence de collusion, qu'elle soit interne ou externe. Dans la mesure où deux personnes ou plus décident de comploter pour commettre une fraude ou une malversation, il devient difficile, voire impossible, pour un vérificateur de détecter les stratagèmes utilisés, qui peuvent comprendre la falsification de documents ou la négligence volontaire.

- Les ressources financières allouées au vérificateur général sont limitées et fixées par la loi.

Nous sommes convaincus que la vérification indépendante des comptes et affaires de la Ville, telle qu'elle est pratiquée par le Bureau du vérificateur général de Montréal, demeure un moyen important pour s'assurer de la saine gestion des deniers publics. Cependant, en raison des contraintes évoquées précédemment, il serait illusoire d'espérer que le vérificateur général puisse détecter toutes les menaces de fraude et de corruption pouvant survenir dans une grande ville comme Montréal. Nous n'avons ni les pouvoirs ni les ressources d'une commission d'enquête et des autorités policières.

\section{Les vérificateurs généraux impuissants devant la corruption des Administrations?}

Alors que notre lettre appelait à un questionnement des pratiques actuelles pour empêcher ces événements de se reproduire, la réponse des vérificateurs de la Ville de Montréal n'aborde aucunement cette essentielle (et inévitable) remise en question du rôle joué par les auditeurs dans la lutte contre la corruption. Elle s'apparente davantage à un exercice d'autojustification concernant le travail accompli au cours des dernières années. Pourtant, l'enquête en cours est en train de faire la preuve d'une défaillance majeure des contrôles mis en place à la Ville de Montréal. Et les auditeurs font partie (et en sont une constituante fondamentale) de l'environnement de contrôle qui semble avoir failli.

M. Bergeron et ses collègues ont répondu à l'interrogation "Où étaient les vérificateurs? " dans le sens littéral de la question. Ils ont répondu en énumérant les tâches qu'ils ont accomplies pendant que la corruption s'installait inlassablement au cœur même de l'administration municipale dont ils vérifiaient la gestion. Cette réponse est une démonstration on ne peut plus parlante d'une vision " micro " des auditeurs, non seulement de leurs tâches, mais aussi de leur rôle en tant que gardiens de l'Administration. 
Les vérificateurs avancent que la portée de leur travail et leurs conclusions sont limitées par leurs pouvoirs d'enquête qui ne sont pas ceux des autorités policières. Les auditeurs n'ont pas à se livrer à des enquêtes comme telles dans les Administrations; ce n'est pas là ni leur rôle ni leur domaine de compétences. Néanmoins, ils ont le devoir d'évaluer les risques de fraude en présence, et cela, sans relâche. Lorsqu'ils auditent les états financiers, il est obligatoire que les auditeurs réévaluent chaque année les risques de fraude au sein de l'organisation qu'ils vérifient. Les normes de pratique de l'audit des états financiers sont très claires à cet égard ${ }^{3}$ :

A8. Bien qu'on ne puisse s'attendre à ce que l'auditeur fasse totalement abstraction du jugement que son expérience passée auprès de l'entité l'a amené à porter sur l'honnêteté et l'intégrité de la direction et des responsables de la gouvernance de l'entité, il est particulièrement important qu'il fasse preuve d'esprit critique lorsqu'il considère les risques d'anomalies significatives résultant de fraudes, étant donné que les circonstances peuvent avoir changé.

A6. Dans le secteur public, les responsabilités de l'auditeur concernant les fraudes peuvent découler de textes légaux ou réglementaires ou d'autres textes émanant d'une autorité qui sont applicables aux entités du secteur public, ou être spécifiquement définies dans le mandat de l'auditeur. En conséquence, il se peut que les responsabilités de l'auditeur d'une entité du secteur public ne se limitent pas à la prise en considération des risques d'anomalies significatives dans les états financiers, mais qu'elles comportent une obligation plus large de considérer les risques de fraude.

Lorsque les auditeurs effectuent des missions d'audit de performance, l'obligation d'apprécier les risques de fraude en présence n'est pas formellement énoncée dans les normes de pratique, mais ce serait adopter une vision très réductrice du rôle de l'auditeur que de ne pas effectuer cette appréciation parce que les normes ne l'exigent pas formellement.

Tous, qu'ils soient gestionnaires ou élus, devront faire un examen de conscience sur les circonstances ayant engendré cette situation. Il faut souhaiter que ces événements susciteront aussi chez les auditeurs des questionnements véritables quant à leur rôle au regard de la prévention et de la détection de la corruption dans les Administrations.

L'heure n'est plus à la justification du travail effectué dans le passé non plus qu'à la résilience pour les vérificateurs généraux se disant limités dans la portée et l'étendue de leur travail d'audit des Administrations. Si les auditeurs n'ont pas suffisamment de pouvoirs ou de ressources pour assumer pleinement leur rôle de gardiens, qu'ils en fassent la démonstration aux autorités, et si les autorités restent sourdes à leur requête, ils auront toujours la possibilité de rendre public le refus des autorités de leur allouer les pouvoirs et les ressources demandés.

3 Norme canadienne d'audit - NCA 240 - Responsabilités de l'auditeur concernant les fraudes lors d'un audit d'états financiers, paragraphes A8 et A6 (Collection Normes et recommandations de I'ICCA). 
Les vérificateurs généraux disposent d'une position privilégiée en raison de leur statut institutionnel. Il y a des limites visibles à ce qu'ils peuvent faire pour assumer pleinement leur rôle de gardiens des Administrations, et espérons que les autorités sauront y remédier. Mais il y a aussi les limites invisibles, celles que les auditeurs s'imposent à eux-mêmes lorsqu'ils exécutent leur travail d'audit. Les vérificateurs généraux risquent de devenir (ou d'être vus comme) des tigres de papier s'ils persistent à justifier la pertinence de leur travail alors que preuve est faite que les cibles qu'ils ont visées n'étaient pas les bonnes, que les lacunes qu'ils ont identifiées en cachaient d'autres bien pires, que les recommandations qu'ils ont émises ont été suivies à moitié et que les alarmes qu'ils ont sonnées n'ont pas été entendues ou écoutées.

\section{- CONCLUSION : « FAIRE SAVOIR »}

L'anthropologue Michael Taussig définit la notion de « secret public " comme étant ce qui est connu de tous, mais qui ne peut être articulé et énoncé publiquement (Taussig, 1999). Le secret public entourant certaines choses est parfois tel que même l'apparence de savoir ledit secret doit être soigneusement évitée et dissimulée : " [people] know they must not know " (Taussig, 1999, p. 131). C'est à partir de son expérience dans la Colombie déchirée des années 1980 que Taussig développe la notion de secret public :

We all "knew" this [collusion between death squads and the military], and they "knew" we "knew," but there was no way it could be easily articulated, certainly not on the ground, faceto-face. Such "smoke screens" are surely long known to mankind, but this "long knowness" is itself an intrinsic part of knowing what not to know [. . . Such is the labor of the negative, as when it is pointed out that something may be obvious, but needs stating in order to be obvious. For example, the public secret. Knowing it is essential to its power, equal to its denial (Taussig, 1999, p. 6).

L'expérience colombienne de Taussig et son concept de " secret public » résonnent étrangement dans le contexte des révélations de la commission Charbonneau indiquant que les pratiques mafieuses de collusion et de corruption étaient apparemment connues de (presque) tous au sein de l'administration de la Ville de Montréal. Comme si tout le monde savait, mais en même temps, chacun savait qu'il ne devait pas savoir. Bref, la loi du silence semble s'être insidieusement installée à l'hôtel de ville. En mobilisant les travaux de Taussig, Vaughan Radcliffe (2008, p. 106-107) fait la remarque suivante à propos de la vérification dans le secteur public :

In its amenability and ready adherence to the public secrets of modern society government auditing does in fact tend towards an attendance to those in power, though this may be unintended, through its provision of a reliable stream of managerial diagnoses and an abrogation of political debate, and this is in itself a significant factor in the rapid expansion of auditing in government [...] I believe that a careful reading of the products of audit inquiry provides evidence of the complicity of auditing in maintaining public secrets [...] In just one example of this, and as has been said elsewhere, the language of auditing is itself defining, facilitating, for example, the definition of political problems as business problems, and hence displacing political debate of matters that could potentially prove embarrassing. 
Ce que nous rappelle Radcliffe avec toute la profondeur empirique de ses propres travaux de recherche, c'est que la mission d'un vérificateur général n'est pas simplement de savoir, mais également de " faire savoir ". Faire savoir " publiquement » n'est pas qu'une question d'indépendance. Elle implique également de la part du vérificateur général de considérer soigneusement le choix des mots pour qualifier la nature des dysfonctionnements qu'il démasque et alerter avec justesse le public. Lorsque l'origine de ces dysfonctionnements est de nature politique, ils doivent être décrits et caractérisés comme tels. Autrement dit, les missions d'audit de performance ne peuvent se limiter à une appréciation technique des systèmes de gestion vérifiés. Les vérificateurs généraux ne sont pas simplement des techniciens, ils ont également une responsabilité politique à assumer pour faire émerger la vérité et rendre visibles les secrets publics et politiques des Administrations. Cette responsabilité ne dépend pas uniquement des vérificateurs généraux. Elle incombe également aux chercheurs universitaires qui par leurs travaux doivent contribuer à identifier les défis de la vérification dans le secteur public et à renforcer de façon constructive la légitimité des vérificateurs généraux au service des citoyens.

\section{BIBLIOGRAPHIE}

Arendt, H. (1979). On Revolution, New York, Penguin Books.

Cooper, C. (2005). « Accounting for the Public Interest: Public Ineffectuals or Public Intellectuals ", Accounting, Auditing and Accountability Journal, vol. 18, n 5, p. 592-607.

Dunleavy, P. et C. C. Hood (1994). "From Old Public Administration to New Public Management ", Public Money and Management, vol. 14, n² 2, p. 9-16.

Malsch, B. et H. Guénin-Paracini (à paraître). " The Moral Potential of Instrumental Reason in Accounting Research ", Critical Perspectives on Accounting.

Morin, D. (2002). Influence of Value for Money Audit on Public Administrations: Looking Beyond Appearances, Financial Accountability \& Management, vol. 17, $\mathrm{n}^{\circ} 2$, p. 99-117.

Neu, D., D. J. Cooper et J. Everett (2001). "Critical Accounting Interventions ", Critical Perspectives on Accounting, vol. 12, $\mathrm{n}^{\circ}$ 6, p. 735-762.

Radcliffe, V. S. (2008). «Public Secrecy in Auditing: What Government Auditors cannot Know ", Critical Perspectives on Accounting, vol. 19, n 1, p. 99-126.

Taussig, M. (1999). Defacement: Public Secrecy and the Labor of the Negative, Stanford, Stanford University Press. 